\title{
MYB transcription factor CiMYB42 regulates limonoids biosynthesis in citrus
}

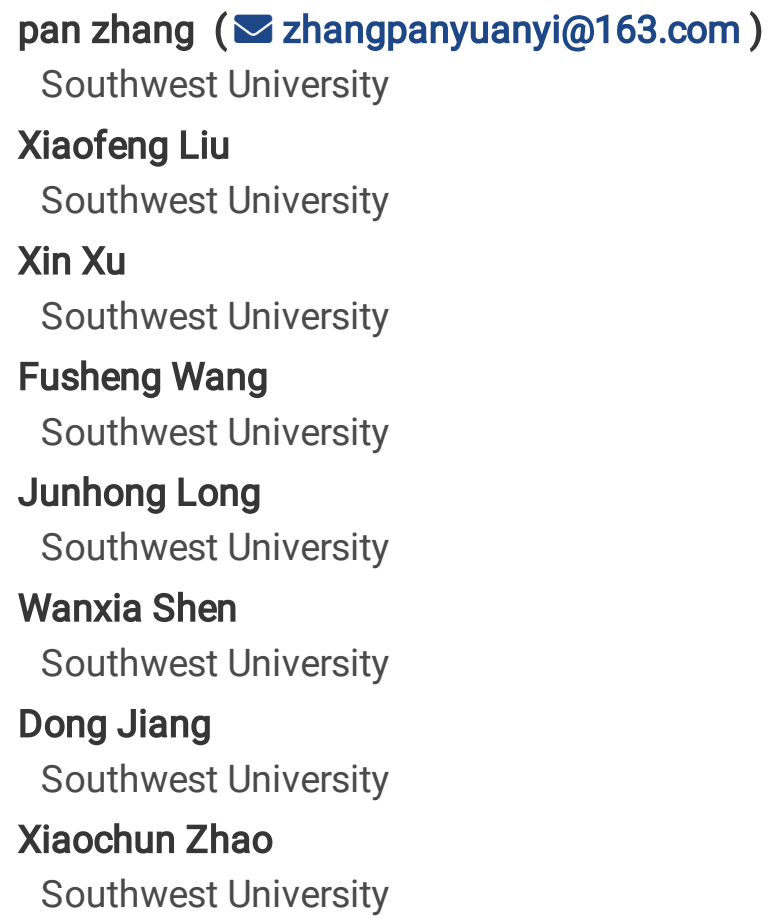

\section{Research article}

Keywords: limonoids biosynthesis, R2R3MYB, CiMYB42, CiOSC, triterpenoid

Posted Date: January 22nd, 2020

DOI: https://doi.org/10.21203/rs.2.21480/v1

License: (c) (1) This work is licensed under a Creative Commons Attribution 4.0 International License. Read Full License

Version of Record: A version of this preprint was published at BMC Plant Biology on June 3rd, 2020. See the published version at https://doi.org/10.1186/s12870-020-02475-4. 


\section{Abstract}

Backgrounds Limonoids, a major bioactive components, are produced by triterpenoids metabolic pathway. So far, the detailed biochemical reactions regarding to limonoid biosynthesis and their molecular regulation remain elusive. The identification of transcription factors that regulate limonoids biosynthetic pathways is not only necessary for understanding the regulatory mechanisms but also as a tool for manipulating biosynthetic genes for biotechnological applications.

Results In this study, CiMYB42 transcription factor was isolated and identified. Multiple alignment analysis and phylogenetic analysis demonstrated that CiMYB42 is a typical R2R3MYB transcription factor and its amino acid is similar to that of AtMYB42. The limonoids content was higher in the citrus sinensis and citrus grandis than other species. The diverse accumulation patterns also showed in different leaf developmental stages. Expression of CiMYB42 was significantly related to limonoids content and the expression of CiOSC in some of citrus varieties. CiMYB42 transgenic sweet orange resulted significantly change on limonoids contents. Noticeably, CiMYB42 -RNAi induced dwarf phenotype and mainly decreased nomilin accumulation. Overexpressing CiMYB42 mainly increased limonin content. Yeast one hybrid assay results indicated that CiMYB42 exclusively bind to the promoter of CiOSC. In brief, CiMYB42 involved in the limonoids biosynthesis through binding the promoter of CiOSC in citrus.

Conclusions These results indicated that CiMYB42 is an important transcription activator involved in limonoids biosynthesis by regulating the expression of CiOSC. This is the first report elucidating the role of transcription factor in citrus limonoids biosynthesis. Our contributions will provide a reference to understanding regulatory mechanisms of R2R3MYB TFs in the triterpenoids biosynthetic pathway.

\section{Background}

Citrus is one of the most important fruit crops in the world. Citrus produces diverse secondary metabolites, and one of those is limonoids. limonoids has extensive biological and pharmacological activities [1], such as antioxidant [2] and insect antifeedant [3,4] as well as antibacterial [5, 6], anticancer [7-9], antiviral [10, 11], anti-inflammatory [12]. Production of limonoids are varied in different citrus species, different organs and tissues, and different development stages [13-15].

Limonoids, as tetracyclic triterpene compounds, were synthesized from the beginning of isopentenyl diphosphate (IPP) and dimethylallyl diphosphate (DMAPP), which are produced by mevalonate (MVA) pathway and methylerythritol phosphate (MEP) pathway $[16,17]$. Condensation of IPP and DMAPP forms C15 farnesyl diphosphate (FPP), which is further catalyzed by squalene synthase (SQS) in head to head condensation to form the linear C30 triterpenoid precursor, squalene. Subsequently, squalene epoxidase (SQE) oxidates squalene to form 2, 3oxidosqualene, which undergoes cyclization by specific oxidosqualene cyclases (OSCs) to form diverse triterpenoids skeletons [18]. Squalene is the first precursor of triterpenoids such as limonoids, sterols, and brassinosteroids. SQS plays an important regulatory role because it locates in a key branch point and acts as a switch [19]. SQE and OSC are the key rate-limiting enzymes in triterpenoid biosynthesis, catalyzing the first oxygenation and cyclization step, respectively $[20,21]$. Strategies to change the triterpenoids production by manipulating genes that code for triterpenoids pathway enzymes have been deeply reported [22-25]. Besides, transcription factors (TFs) holds great potential for improving production of the secondary metabolites by activating or repressing structural genes in metabolic pathway by binding to their promoter regions [26]. Hence, they are ideal targets for manipulating the production of triterpenoids. 
None of the TFs has been reported from citrus that regulates triterpenoids production, but several TFs from other plants involved in triterpenoids biosynthesis have been identified. The most impressive experiment was conducted by Shang et.al. [27]. In cucumber (Cucumis sativus), bHLH TFs of BI (Bitter leaf) and Bt (Bitter fruit) could bind to the promoter of $\mathrm{Bi}$ (the member of OSC) to regulate the biosynthesis of cucurbitacin C. TRITERPENE SAPONIN BIOSYNTHESIS ACTIVATING REGULATOR1 (TSAR1) and TSAR2 are two homologous jasmonate-inducible bHLH transcription factors, which directly influence triterpene saponin biosynthesis by interacting with the promoter of 3HYDROXY-3-METHYLGLUTARYLCOENZYME A REDUCTASE1 (HMGR1) and MAKIBISHI1 in Medicago truncatula [28]. Licorice (Glycyrrhiza uralensis) bHLH TF, GubHLH3, positively regulates the expression of soyasaponin biosynthetic genes [29]. Recently, WsWRKY1 of Withania. somnifera also shows direct regulation of triterpenoid pathway by binding to W-box sequences in promoters of SQS and SQE [30]. Besides, MYB TFs are also crucial regulators participated in plant terpenoids metabolism. Overexpression of grapevine (Vitis vinifera) VvMYB5b in tomato induced interesting effects, which phenylpropanoid metabolism and beta-amyrin was down regulated whereas beta-carotene was up regulated [31]. The P. taeda PtMYB14 was also likely related to phenylpropanoid biosynthesis and terpenoids biosynthesis [32, 33]. Both PtMYB14 and VvMYB5b are the members of R2R3-MYBs, which are likely to regulate terpenoids biosynthesis. Recently, another R2R3-MYB member, SmMYB36 from Salvia miltiorrhiza Bunge also inhibited the phenylpropanoid biosynthesis (phenolic acid) and promoted the accumulation of diterpenoids (tanshinone) [34].

R2R3MYB transcription factors is one of the largest families of plant TFs. The members of this family extensively participated in terpenoids biosynthesis, not only in triterpenoids, but also other terpenoids. For example, spearmint (Mentha spicata) MsMYB binds to cis-elements of MsGPPS.LSU and suppresses monoterpene biosynthesis [35]. Artemisia annua AaMYB1 acts as activator in diterpene metabolism (artemisinin, AN) [36]. Despite the disclosure of R2R3MYBs in plant terpenoids biosynthesis, their roles in triterpenoids metabolism is still poorly understood, especially in limonoids biosynthesis.

In our previous study, ciclev10021695m, a MYB family TF was found to be related to the biosynthesis of limonoids by RNA-seq analysis [20]. In this study, the role of CiMYB42 was investigated in order to elucidate the regulatory mechanism of CiMYB42 in biosynthesis of limonoids.

\section{Results}

Characteristics of CiMYB42

Genomic sequences of ciclev10021695m was isolated from C. clementina genomic database. It encodes 267 amino acids and has a theoretical isoelectric point and molecular weight of 5.14 and $30.21 \mathrm{kDa}$, respectively.

Ciclev10021695m is an R2R3 MYB transcription factor because it contains a typical conserved R2R3 MYB domain. The amino acid sequence of ciclev10021695m shared a high similarity with AtMYB42 of Arabidopsis (55.86\%), therefore it was accordingly named CiMYB42 (Fig. 1a \&1b).

Phylogenetic analysis of CiMYB42 with other R2R3-MYB genes from Arabidopsis revealed that CiMYB42 is close to the AtMYB42 and AtMYB85, which are also related to the plant secondary metabolism [37]. In addition, our previous work demonstrated that expression level of CiMYB42 significantly related to limonoids content in pummelo seeds [20]. Thus, it suggested that CiMYB42 could be an important regulatory factor in limonoid biosynthesis.

\section{Accumulation of limonoids during leaf development}


Limonin and nomilin are the main components of limonoids. Limonoids contents were varying in different citrus varieties and different leaf development stages (Fig. 2). The contents of limonoids ranged from $0.02 \mathrm{mg} / \mathrm{g}$ FW to $0.53 \mathrm{mg} / \mathrm{g}$ FW in nine different varieties. The highest limonoids content was 26 times greater than that of the lowest one. The variation of limonoids content in different citrus species was also observed. The sweet orange (C. sinensis) presented the highest limonoids content $(0.25-0.53 \mathrm{mg} / \mathrm{g} \mathrm{FW})$, followed by C. grandis $(0.15-0.22 \mathrm{mg} / \mathrm{g} \mathrm{FW})$. However, lomonoids content remained at a quite low level in C. reticulate and F. classifolia, especially in F. classifolia. It suggests that genetic background is one of the most important factors influencing the production of limonoids. In addition, the limonoids content at different leaf development stages also exhibited different accumulation patterns in different species. Three major trends were showed in Fig. 2.

Correlation between CiMYB42 expression and limonoids content

The relative expression of CiMYB42 demonstrated significant differences among the species and leaf development stages. The highest expression level (8.17) was observed in ZSXLMY at Y2 stage, while the lowest expression level (0.70) was found in Y2 stage of GC with 12 folds lower of expression (Fig. 3). The expression level of CiMYB42 also showed three different patterns like those of limonoids content. Significant positive correlations between the level of CiMYB42 expression and limonoids content during leaf development were found in some of varieties (Table 1). However, the correlation was not consistent in all of varieties. Some of them even showed strong negative correlations. Positive correlations between CiMYB42 expression and limonoids content among varieties were found at all three leaf development stages, with much strong correlations at late stages of development $(0.236,0.639$ and 0.66 , respectively). Similar correlations were also observed between limonoids content and CiOSC expression. However, expression of CiMYB42 demonstrated significant correlation with CiOSC during leaf development in most of varieties except GC. Close correlation was also observed among varieties in all three development stages (0.971, 0.824 and 0.81 , respectively).

Table 1

Correlations between CiMYB42 expression and limonoids content

\begin{tabular}{|c|c|c|c|c|c|c|c|c|c|}
\hline $\begin{array}{l}\text { Pearson } \\
\text { Correlation } \\
\text { Coefficient }\end{array}$ & JC & NHE & WCZPG & ST & GC & HPJG & RAJG & GXSTY & ZSXLMY \\
\hline $\begin{array}{l}\text { CiMYB42\& } \\
\text { Limonoids }\end{array}$ & $0.78^{\star}$ & -0.155 & $0.835^{\star \star}$ & 0.615 & $0.691^{*}$ & $-0.768^{*}$ & -0.25 & $-0.781^{\star}$ & $-0.666^{\star}$ \\
\hline $\begin{array}{l}\text { CiOSC\& } \\
\text { Limonoids }\end{array}$ & $0.737^{\star}$ & -0.321 & $0.885^{\star \star}$ & 0.575 & $-0.736^{*}$ & $-0.701^{*}$ & -0.382 & -0.359 & $-0.703^{*}$ \\
\hline $\begin{array}{l}\text { CiMYB42\& } \\
\text { CiOSC }\end{array}$ & $0.943^{\star \star}$ & $0.942^{\star \star}$ & $0.689 *$ & $0.963^{\star \star}$ & $-0.844^{\star \star}$ & $0.855^{\star \star}$ & 0.964 ** & $0.708^{* *}$ & $0.998^{\star \star}$ \\
\hline > > 0.05; & D.01. & & & & & & & & \\
\hline
\end{tabular}

Effect of CiMYB42 on limonoids production in transgenic plants

Overexpression and RNA interference of CiMYB42 were conducted to further elucidate the role of CiMYB42 in limonoids biosynthesis. The recombinant vectors of overexpression and RNA interference were shown in Fig. $4 a$ \& Fig. 4b. The non-transgenic Wanjincheng sweet orange was grafted on same rootstock as those of transgenic plants and used as control. We obtained three CiMYB42 overexpression (CiMYB42-OE) lines and three CiMYB42 RNA 
interference (CiMYB42-R) lines (Fig. 4c \& Fig. 4d). No morphological differences were observed between the control and overexpression lines, but RNAi lines exhibited dwarfing and shorter internodes characters (Fig. 4d).

Subsequently, real-time qPCR was performed to evaluate the expression levels of CiMYB42, CiSQS and CiOSC in transgenic lines and control (Fig. 4f). CiMYB42 expression was significantly higher in overexpression lines than that of in control, especially in overexpression line 1 and 3, which have approximately 6.6 times higher than that of control. Three RNAi transgenic lines (R-1, R-2 and R-3) showed different lower levels of CiMYB42 expression, the line 1, line 2 and 3 was decreased by 39\%, 67\% and 72\%, respectively. The expression of CiSQS was not suppressed by silencing of CiMYB42 in RNAi plants, also not upregulated in overexpression plants (Fig. 4g). Correlation between expressions of those two genes was significantly negative. In contrast, the expression of CiOSC was consistent with the expression of CiMYB42 in transgenic plants.

The change expression of CiOSC and CiMYB42 altered limonoids accumulation, the limonoids content increased by $16.08 \%$ in overexpression lines, the RNAi lines decreased by $22.09 \%$ (Fig. 4g). Noticeably, transgenic CiMYB42 had different effect on the limonin and nomilin content. Limonin content increased by $50 \%$ in overexpression plants, and there was no significant difference between RNAi lines and control. However, nomilin exhibited a great decline in RNAi lines, especially in line 2 (decreased by 48.76\%). In overexpression lines, nomilin content only showed slightly increase (3.31\%). That is to say, overexpression CiMYB42 mainly increased limonin content, while RNAi CiMYB42 mostly decreased nomilin content.

Correlation analysis indicated that limonoids contents significantly correlated to the expressions of CiOSC and CiMYB42. The Pearson correlation coefficient was $0.931(P<0.01)$ and $0.824(P<0.05)$ respectively, but had negatively correlated to the expression of CiSQS.

CiMYB42 regulates limonoid biosynthesis via binding to the promoter of CiOSC

The significant correlations between CiMYB42 and CiOSC, CiSQS demonstrated the possible interaction between them. Thus, the cis-elements of CiOSC and CiSQS promoters were identified by PLANTCARE and PLACE online software. Several conserved cis-elements were found, especially for TF binding sites (Fig. S2). These TF binding sites also play other roles in plant growth and development, like responding to drought and salinity (MYB core), responding to light and abscisic acid (G-box/ MYC, bHLH binding site) and responding to pathogen (W-box, WRKY binding site). The approximately $2 \mathrm{~Kb}$ promoters of CiOSC and CiSQS have been found to contain several MYB core and AC-element, previously reported to be required for MYB binding [38]. On the basis of these cis-elements analysis, Y1H assay was carried out to identify potential interaction between CiMYB42 and promoters of CiOSC and CiSQS. The minimal AbA inhibitory concentration of bait vector were detected as shown in Fig. S3. The results indicated that CiMYB42 directly and exclusively interacted with CiOSC promoter under the suppression of $500 \mathrm{ng} / \mathrm{mL} \mathrm{AbA}$ (Fig. 5). In contrast, there is no interaction between CiMYB42 and CiSQS promoter in yeast cells. This suggests that CiMYB42 acted as an activator regulate the expression of CiOSC. These results indicated that CiMYB42 is one of key factors involving in the biosynthesis of limonoids through regulating the expression of CiOSC.

\section{Discussion}

In this study, CiMYB42 gene was identified to significantly affect the limonoids biosynthesis by regulating the expression of CiOSC. Previous report indicated that CiMYB42 (ciclev10021695m) is a R2R3MYB gene located on scaffold S00271 of sweet orange and S3 of clementina. Its expression could be induced by cold stress, ABA and MeJA treatments [39], but the function was unknown. In general, R2R3MYB transcription factors play important regulatory roles in terpenoids biosynthesis, especially for the members in subgroup 4, 5, 15 [33, 34]. CiMYB42 was 
classified in subgroup 12 from previous report [39]. Our study suggested that members of subgroup 12 may also involve in the regulation of terpenoids biosynthesis.

Variations of limonoids contents in different species and development stages of seeds, and fruits in citrus have been reported $[13,15,40,41]$, So far, there is no report on investigation of regulation on accumulation of limonoids during leaf development among different species of citrus. In this study, limonoids contents were significantly different among different leaf development stages in most of varieties. The trends of limonoids content during leaf development presented several patterns depending on the different genetic backgrounds. The expression of CiMYB42 was not positively correlated to the limonoids contents in most of varieties during leaf development in this study (Table 1). However, similar correlations were also observed between expression of CiOSC and limonoids contents. This may due to CiOSC indirectly participated in limonoids biosynthetic pathway through regulating the synthesis of triterpene [42]. Furthermore, limonoids accumulation is also not only affected by the biosynthesis, but also by other factors such as transportation and degradation.

Reverse genetics approach was applied to determine the role of CiMYB42 in limonoids biosynthesis. Some interesting phenotype were discovered in CiMYB42 silenced sweet orange plants (Fig. 4d). RNAi of CiMYB42 resulted in stunted growth and shorter internodes of the plant with significant decrease in limonoids content. The morphogenetic inhibition and reduced limonoids content caused by silence of CiMYB42 were similar to previous studies in which down-regulation/silence of triterpenoids pathway genes and triterpenoids metabolism related TFs such as NtCAS1 [43], AtHMGR1 [44] and WsWRKY1 [30]. In transgenic plants, it is noticeable that nomilin showed a large decline in RNAi lines. On the one hand, we hypothesized that the effect of CiMYB42 RNAi on nomilin content in sweet orange could be a result of the depletion of limonoids precursors, which are required for limonoids formation [45]. On the other hand, down-regulation of CiOSC expression also could induce nomilin reduction. However, little change of limonin probably because there is still sufficient nomilin supply for limonin synthesis despite it drops dramatically. Indeed, CiMYB42-RNAi leaves exhibited significant down-regulation of CiOSC, which is the first ratelimiting step of triterpene cyclization. Whereas, the CiSQS was slightly up-regulated by CiMYB42-RNAi. Such differential regulation of the triterpenoid pathway has recently been reported in W. somnifera by the WsWRKY1 [30], in tomato by the GAME9 [46], in birch (Betula platyphylla Suk.) by the BpbHLH9 [47].

In contrast to CiMYB42-RNAi, overexpression of CiMYB42 in sweet orange leaves enhanced expression level of CiOSC and limonoids content. This supported the observation made in RNAi studies that CiMYB42 positively regulates CiOSC expression and limonoids content. This observation was consolidated by $\mathrm{Y} 1 \mathrm{H}$ assay, which indicated that CiMYB42 exclusively activated the promoter of CiOSC. And it is interesting that the increased limonoids mainly are limonin accumulation instead of nomilin. This suggest that CiMYB42 have multiple functions: in addition to activating the expression of CiOSC directly, it may could induce the expression of other downstream genes to accumulate limonin. In general, one target genes were regulated by several TFs, in the same way, one TF also involved in multiple biosynthetic step. Such result was also reported by previous observations $[48,49]$. The fact that CiMYB42-transgenics effectively altered limonoids accumulation give us a better understanding of the molecular mechanisms that control the citrus limonoids biosynthesis. It will be important for future commercial biopharming applications and citrus processing industry.

The comparative transcriptomic analysis was conducted with transgenic plants to illustrate other genes associated with CiMYB42 (details of data not shown.). Altered expression of CiMYB42, particularly silenced expression led to the significantly change on gene expression involved many secondary metabolic process. Besides the sequiterpenoid and triterpenoid biosynthesis, phenylpropanoid metabolism are also significantly affected. 
Flavonoids biosynthesis related pathways are the main groups of secondary metabolism affected by the CiMYB42 in transgenic plants. Recent studies also revealed that some members of MYB transcription factors family participate in both flavonoids and terpenoids biosynthetic pathways [31, 34]. Thus, CiMYB42 not only regulates triterpenoids biosynthetic pathway, but also have potential influences other secondary metabolism.

\section{Conclusions}

In this study, we identified a novel regulatory factor, CiMYB42, which involved in limonoids biosynthesis by binding to the promoter of CiOSC. The results indicated that CiMYB42 is a transcription activator in limonoids metabolic network. This work will provide new insight into the regulatory network of limonoids biosynthesis in citrus and construct a basis for changing limonoids content by transgenic technology.

\section{Materials And Methods}

\section{Plant materials and sampling}

In April 2017, health and fresh leaves in three different stages (Y1, Y2 and Y3) and different species were collected from the National Citrus Germplasm Repository, located in the Citrus Research Institute of Chinese Academy of Agricultural Sciences (CRIC), in Beibei, Chongqing, China. These samples were used for both gene expression analysis and determination of limonoids content. The leaf samples at three developmental stages were showed in Fig. S1. Citrus samples used in this study were shown in Table 2.

Table 2

Citrus samples used in this study

\begin{tabular}{|lll|}
\hline Name of species & Name of varieties & Abbreviation used in this study \\
\hline Citrus sinensis (L.) Osbeck & Beibei 447 orange & JC \\
\hline Citrus sinensis (L.) Osbeck & Newhall navel orange & NHE \\
\hline Citrus grandis (L.) Osbeck & Guangxi Shatianyou pummelo & GXSTY \\
\hline Citrus grandis (L.) Osbeck & Early Siam pummelo & ZSXLMY \\
\hline Citrus reticulata Blanco & Morita Unshu mandarin & ST \\
\hline Citrus reticulata Blanco & Miyagawa Wase mandarin & GC \\
\hline Citrus reticulata Blanco & Wangcang Zoupigan mandarin & WCZPG \\
\hline Fortunella classifolia Swingle & Huapi kumquat & HPJG \\
\hline Fortunella classifolia Swingle & Rongan kumquat & RAJG \\
\hline
\end{tabular}

Extraction and determination of limonin and nomilin

The extraction and determination of limonin and nomilin with HPLC were assayed according to the method described by Sun et al.[13], and three biological replicates were performed in this experiment. Limonoids content in this study is the sum of limonin and nomilin contents.

Bioinformation analysis of CiMYB42 
The amino acid sequence of CiMYB42 was obtained from $\mathrm{C}$. clementina genome database. The theoretical PI (isoelectric point) and MW (molecular weight) were predicted by EXPASY (https://web.expasy.org/compute_pi/). Simple modular architecture research tool (SMART) was used to confirm the domain of CiMYB42. The protein sequences were blasted to the Phytozome database to identify homologous sequences in other plant species. DNAMAN v.6.0 was used for multiple alignment analysis. The homologous sequences of Arabidopsis were used for construction of phylogenetic tree.

\section{DNA and RNA extraction, cDNA synthesis and relative expression analysis}

Genomic DNA was extracted by CTAB method, RNA was extracted using the RNAprep pure plant Kit following the manufacturer's instructions (Tiangen biotech, Beijing, China). RNA $(1 \mu \mathrm{g})$ was reverse transcribed into cDNA using PrimeScript 1st Strand cDNA Synthesis Kit with gDNA Eraser (Perfect Real Time) (Takara Biomedical Technology, Beijing, China). Detection of gene expression was performed by Real-time qPCR using $1 \times \mathrm{iTaq}^{\mathrm{TM}}$ universal SYBR $^{\circ}$ Green Supermix (Bio-Rad). The primers used are listed in supplementary Table. S1. Experiments were repeated three times, using citrus Actin gene for normalization, the relative expression levels were calculated using $2^{-\Delta \Delta C t}$ method [51].

\section{Transformation and characterization of transgenic sweet orange}

The CDS of CiMYB42 was amplified and ligated into Sac I/BamH I sites of pBI121CSH to obtain the overexpression vector. For construction of RNAi vector, a 462 bp fragment was PCR amplified and integrated into pGBi vector. GFP was used as report gene (Fig. 4e). The constructs were verified by sequencing and transformed into Agrobacterium tumefaciens strain EHA105 by electric shock. The overexpression vector and RNAi vector were transformed into Wanjincheng orange epicotyl explants as previously reported [52]. The transformants were selected on MS medium containing $50 \mathrm{mg} / \mathrm{ml}$ kanamycin. The positive shoots were confirmed by GFP-fluorescent detection and genomic DNA PCR. The GFP-positive shoots were grafted onto rootstocks of two years old Ziyang Xiangcheng (C. junos). Integration and expression of the CiMYB42 gene in transformed shoots were further confirmed by RT-PCR analysis.

\section{Yeast one-hybrid (Y1H) assay}

The full-length CiMYB42 ORF was amplified and fused to pGADT7 vector to create the prey. The $2 \mathrm{~Kb}$ promoters of CiSQS (ciclev10028537m) and CiOSC (ciclev10010416m) were synthesized by Beijing Genomics Institute and inserted into pAbAi vector as the bait. The primers used for vector construction are listed in Table. S1. Yeast onehybrid $(\mathrm{Y} 1 \mathrm{H})$ assays were performed using the Matchmaker Gold Yeast One-Hybrid System (Clontech, USA). pAbAiSQS and pAbAi-OSC were linearized by Bbsl enzyme and minimal inhibitory concentration of aureobasidin A (AbA) were detected on SD/-Ura medium. Interactions between CiMYB42 and the promoters of CiSQS and CiOSC were subsequently tested by co-transformation of linearized bait plasmid and prey plasmid into Y1H Gold yeast competent cells on SD/-leu medium with optimal AbA concentration.

\section{Statistical analysis}

The statistical analysis was performed using SPSSV20.0 statistical package. Significant differences were subjected to a Duncan's test. $\mathrm{P}<0.05$ was considered significant. The correlation analysis was conducted through Pearson's correlation analysis.

\section{Declarations}

\section{Ethics approval and consent to participate}


Not applicable.

\section{Consent for publication}

Not applicable.

\section{Availability of data and materials}

The accession numbers of the CiMYB42, CiSQS and CiOSC can be found in the Phytozome. All data analyzed in this study are included in this published article and its supplementary files.

\section{Competing interests}

The authors declare that they have no competing interests.

\section{Funding}

This study was supported by the "Double World-classes" Development Plan of Southwest University, which provides us a good research platform. The National Key R\&D Program of China (2019YFD1001401-02); the Ministry of Education double first-class discipline construction project "Breeding and Application of new crop variety and germplasm”, the Earmarked Fund for Chongqing Special \& Economic Agriculture Research System on Late Maturation Citrus and the Earmarked Fund for China Agriculture Research System (CARS-26) make a great contribution to sample selection and sweet orange transformation system. Foundation for the Talents/Doctor by Southwest University (SWU117038) and Chongqing Research Project of Foundations and Frontiers (cstc2018jcyjAX0400) provides us financial support with the experiment of sequencing analysis.

\section{Authors' contributions}

$\mathrm{PZ}$ and $\mathrm{X}-\mathrm{CZ}$ designed the experiments; $\mathrm{PZ}$ conducted the experiment and prepared the manuscript; $\mathrm{X}-\mathrm{CZ}$ and $\mathrm{XY}$ revised the manuscript. $X-F L$ provided the vector and scientific guidance of the sweet orange transformation; $X Y$ provided useful suggestion of data collection and analysis. F-SW helped with the sample preparation and limonoids determination; J-HL helped with the yeast one hybrid assay; W-XS helped with the qPCR experiment; DJ conducted sample selection and corrected sample name. All authors have read and approved the manuscript for publication.

\section{Acknowledgements}

The authors greatly acknowledge Aihong Peng at Citrus Research Institute of Southwest University for providing useful supervision on sweet orange transformation.

\section{Abbreviations}

IPP

isopentenyl diphosphate; DMAPP:dimethylallyl diphosphate; MVA:mevalonate; MEP:methylerythritol phosphate; FPP:farnesyl diphosphate; SQS:squalene synthase; SQE:squalene epoxidase; OSC:oxidosqualene cyclases;

TF:transcription factor; CDS:coding sequence; ORF:open reading frame; JC:Beibei 447 Jingcheng; NHE:Newhall navel orange; GXSTY:Guangxi Shatian pummelo; ZSXLMY:Early Siam pummelo; ST:Shengtian Satsuma Mandarin; GC:Gongchuan Satsuma Mandarin, WCZPG:Wangcang Zoupi Gan; HPJG:Huapi kumquat; RAJG:Rongan kumquat; SD:synthetic dropout; Ura:uracil; Leu:leucine; AbA:Aureobasidin A. 


\section{References}

1.

Gualdani R, Cavalluzzi M, Lentini G, Habtemariam S. The chemistry and pharmacology of citrus limonoids. Molecules. 2016;21(11):1530.

2.

Qin S, Lv C, Wang Q, Zheng Z, Sun X, Tang M, Deng F. Extraction, identification, and antioxidant property evaluation of limonin from pummelo seeds. Animal Nutrition. 2018;4(3):281-7.

3.

Roy A, Saraf S. Limonoids: overview of significant bioactive triterpenes distributed in plants kingdom. Biol Pharm Bull. 2006;29(2):191-201.

4.

Vásquez-Rivera A, Chicaiza-Finley D, Hoyos RA, Orozco-Sánchez F. Production of limonoids with insect antifeedant activity in a two-stage bioreactor process with cell suspension culture of azadirachta indica. Appl Biochem Biotech. 2015;177(2):334-45.

5.

Fan X, Lin P, Lu X, Zi J. A new spiro-type limonoid from Azadirachta indica A. Juss. Tetrahedron Lett. 2019;60(16):1158-60.

6.

Maneerat W, Laphookhieo S, Koysomboon S, Chantrapromma K. Antimalarial, antimycobacterial and cytotoxic limonoids from Chisocheton siamensis. Phytomedicine. 2008;15(12):1130-4.

7.

Chen J, Fan X, Zhu J, Song L, Li Z, Lin F, Yu R, Xu H, Zi J. Limonoids from seeds of Azadirachta indica A. Juss. and their cytotoxic activity. Acta Pharm Sin B. 2018;8(4):639-44.

8.

Chidambara Murthy KN, Jayaprakasha GK, Patil BS. Obacunone and obacunone glucoside inhibit human colon cancer (SW480) cells by the induction of apoptosis. Food Chem Toxicol. 2011;49(7):1616-25.

9.

El-Readi MZ, Hamdan D, Farrag N, El-Shazly A, Wink M. Inhibition of P-glycoprotein activity by limonin and other secondary metabolites from Citrus species in human colon and leukaemia cell lines. Eur J Pharmacol. 2010;626(23): $139-45$.

10.

Yan Y, Yuan C, Di Y, Huang T, Fan Y, Ma Y, Zhang J, Hao X. Limonoids from Munronia henryi and their anti-tobacco mosaic virus activity. Fitoterapia. 2015;107:29-35.

11.

Yu J, Wang G, Han Y, Wu Y, Wainberg MA, Yue J. Limonoids with anti-HIV activity from Cipadessa cinerascens. J Nat Prod. 2015;78(6):1243-52.

12.

Hu J, Song Y, Mao X, Wang Z, Zhao Q. Limonoids isolated from Toona sinensis and their radical scavenging, antiinflammatory and cytotoxic activities. J Funct Foods. 2016;20:1-9.

13.

Sun C, Chen K, Chen Y, Chen Q. Contents and antioxidant capacity of limonin and nomilin in different tissues of citrus fruit of four cultivars during fruit growth and maturation. Food Chem. 2005;93(4):599-605.

14.

Page 10/17 
Bauri AK, Foro S, Nhu QND. Limonin isolated from the seeds of Citrus limetta (Indian sweet lemon). IUCrData. 2016; 1(2).

15.

Li S, Wang Z, Ding F, Sun D, Ma Z, Cheng Y, Xu J. Content changes of bitter compounds in 'Guoqing No.1' Satsuma mandarin (Citrus unshiu Marc.) during fruit development of consecutive 3 seasons. Food Chem. 2014;145:963-9. 16.

Vranova E, Coman D, Gruissem W. Network analysis of the MVA and MEP pathways for isoprenoid synthesis. Annu Rev Plant Biol. 2013;64:665-700.

17.

Xu R, Fazio GC, Matsuda SPT. On the origins of triterpenoid skeletal diversity. Phytochemistry. 2004;65(3):261-91. 18.

Pandreka A, Dandekar DS, Haldar S, Uttara V, Vijayshree SG, Mulani FA, Aarthy T, Thulasiram HV. Triterpenoid profiling and functional characterization of the initial genes involved in isoprenoid biosynthesis in neem (Azadirachta indica). Bmc Plant Biol. 2015; 15(1).

19.

Fu J, Liu G, Yang M, Wang X, Chen X, Chen F, Yang Y. Isolation and functional analysis of squalene synthase gene in tea plant Camellia sinensis. Plant Physiol Bioch. 2019;142:53-8.

20.

Wang F, Wang M, Liu X, Xu Y, Zhu S, Shen W, Zhao X. Identification of putative genes involved in limonoids biosynthesis in citrus by comparative transcriptomic analysis. Front Plant Sci. 2017; 8.

21.

Han JY, Jo HJ, Kwon EK, Choi YE. Cloning and characterization of oxidosqualene cyclases involved in taraxasterol, taraxerol and bauerenol triterpene biosynthesis in Taraxacum coreanum. Plant Cell Physiol. 2019;60(7):1595-603. 22.

Jiang D, Rong Q, Chen Y, Yuan Q, Shen Y, Guo J, Yang Y, Zha L, Wu H, Huang L. Molecular cloning and functional analysis of squalene synthase (SS) in Panax notoginseng. Int J Biol Macromol. 2016;95:658.

23.

Dong L, Pollier J, Bassard J, Ntallas G, Almeida A, Lazaridi E, Khakimov B, Arendt P, de Oliveira LS, Lota F, Goossens A, Michoux F, Bak S. Co-expression of squalene epoxidases with triterpene cyclases boosts production of triterpenoids in plants and yeast. Metab Eng. 2018;49:1-12.

24.

Yang Y, Ge F, Sun Y, Liu D, Chen C. Strengthening triterpene saponins biosynthesis by over-expression of farnesyl pyrophosphate synthase gene and RNA interference of cycloartenol synthase gene in Panax notoginseng Cells. Molecules. 2017;22(4):581.

25.

Zheng X, Luo X, Ye G, Chen Y, Ji X, Wen L, Xu Y, Xu H, Zhan R, Chen W. Characterisation of two oxidosqualene cyclases responsible for triterpenoid biosynthesis in Ilex asprella. Int J Mol Sci. 2015;16(2):3564-78.

26.

Zhong R, Lee C, Ye Z. Evolutionary conservation of the transcriptional network regulating secondary cell wall biosynthesis. Trends Plant Sci. 2010;15(11):625-32.

27.

Shang Y, Ma Y, Zhou Y, Zhang H, Duan L, Chen H, Zeng J, Zhou Q, Wang S, Gu W, Liu M, Ren J, Gu X, Zhang S, Wang Y, Yasukawa K, Bouwmeester HJ, Qi X, Zhang Z, Lucas WJ, Huang S. Biosynthesis, regulation, and domestication bitterness in cucumber. Science. 2014;346(6213):1080-4. 
28.

Mertens J, Pollier J, Vanden Bossche R, Lopez-Vidriero I, Franco-Zorrilla JM, Goossens A. The bHLH transcription factors TSAR1 and TSAR2 regulate triterpene saponin biosynthesis in Medicago truncatula. Plant Physiol. 2015;170(1):194-210.

29.

Tamura K, Yoshida K, Hiraoka Y, Sakaguchi D, Chikugo A, Mochida K, Kojoma M, Mitsuda N, Saito K, Muranaka T, Seki $\mathrm{H}$. The basic helix-loop-helix transcription factor GubHLH3 positively regulates soyasaponin biosynthetic genes in Glycyrrhiza uralensis. Plant Cell Physiol. 2018;59(4):783-96.

30 .

Singh AK, Kumar SR, Dwivedi V, Rai A, Pal S, Shasany AK, Nagegowda DA. A WRKY transcription factor from Withania somnifera regulates triterpenoid withanolide accumulation and biotic stress tolerance through modulation of phytosterol and defense pathways. New Phytol. 2017;215(3):1115-31.

31.

Mahjoub A, Hernould M, Joubès J, Decendit A, Mars M, Barrieu F, Hamdi S, Delrot S. Overexpression of a grapevine R2R3-MYB factor in tomato affects vegetative development, flower morphology and flavonoid and terpenoid metabolism. Plant Physiol Bioch. 2009;47(7):551-61.

32.

Bedon F, Levasseur C, Grima-Pettenati J, Séguin A, MacKay J. Sequence analysis and functional characterization of the promoter of the Picea glauca Cinnamyl Alcohol Dehydrogenase gene in transgenic white spruce plants. Plant Cell Rep. 2009;28(5):787-800.

33.

Bedon F, Bomal C, Caron S, Levasseur C, Boyle B, Mansfield SD, Schmidt A, Gershenzon J, Grima-Pettenati J, Séguin A, MacKay J. Subgroup 4 R2R3-MYBs in conifer trees: gene family expansion and contribution to the isoprenoid- and flavonoid-oriented responses. J Exp Bot. 2010;61(14):3847-64.

34.

Ding K, Pei T, Bai Z, Jia Y, Ma P, Liang Z. SmMYB36, a novel R2R3-MYB transcription factor, enhances tanshinone accumulation and decreases phenolic acid content in Salvia miltiorrhiza hairy roots. Sci Rep-Uk. 2017; 7(1).

35.

Reddy VA, Wang Q, Dhar N, Kumar N, Venkatesh PN, Rajan C, Panicker D, Sridhar V, Mao H, Sarojam R. Spearmint R2R3-MYB transcription factor MsMYB negatively regulates monoterpene production and suppresses the expression of geranyl diphosphate synthase large subunit (MsGPPS.LSU). Plant Biotechnol J. 2017;15(9):1105-19.

36.

Matías-Hernández L, Jiang W, Yang K, Tang K, Brodelius PE, Pelaz S. AaMYB1 and its orthologue AtMYB61 affect terpene metabolism and trichome development in Artemisia annua and Arabidopsis thaliana. Plant $\mathrm{J}$. 2017;90(3):520-34.

37.

Zhao K, Bartley LE. Comparative genomic analysis of the R2R3 MYB secondary cell wall regulators of Arabidopsis, poplar, rice, maize, and switchgrass. Bmc Plant Biol. 2014;14(1):135.

38.

Kelemen Z, Sebastian A, Xu W, Grain D, Salsac F, Avon A, Berger N, Tran J, Dubreucq B, Lurin C, Lepiniec L, ContrerasMoreira B, Dubos C. Analysis of the DNA-Binding Activities of the Arabidopsis R2R3-MYB Transcription Factor Family by One-Hybrid Experiments in Yeast. Plos One. 2015;10(10):e141044.

39. 
Xie R, Li Y, He S, Zheng Y, Yi S, Lv Q, Deng L. Genome-wide analysis of citrus R2R3MYB genes and their spatiotemporal expression under stresses and hormone treatments. Plos One. 2014;9(12):e113971.

40.

Dandekar DV, Jayaprakasha GK, Patil BS. Hydrotropic extraction of bioactive limonin from sour orange (Citrus aurantium L.) seeds. Food Chem. 2008;109(3):515-20.

41.

Wang F, Yu X, Liu X, Shen W, Zhu S, Zhao X. Temporal and spatial variations on accumulation of nomilin and limonin in the pummelos. Plant Physiol Bioch. 2016;106:23-9.

42.

Thimmappa R, Geisler K, Louveau T, O'Maille P, Osbourn A. Triterpene biosynthesis in plants. Annu Rev Plant Biol. 2014;65(1):225-57.

43.

Gas-Pascual E, Berna A, Bach TJ, Schaller H. Plant oxidosqualene metabolism: cycloartenol synthase-dependent sterol biosynthesis in Nicotiana benthamiana. Plos One. 2014;9(10):e109156.

44.

Suzuki M, Kamide Y, Nagata N, Seki H, Ohyama K, Kato H, Masuda K, Sato S, Kato T, Tabata S, Yoshida S, Muranaka T. Loss of function of 3-hydroxy-3-methylglutaryl coenzyme A reductase 1 (HMG1) in Arabidopsis leads to dwarfing, early senescence and male sterility, and reduced sterol levels. Plant J. 2004;37:750-61.

45.

Hasegawa S, Herman Z. Biosynthesis of limonoids: Conversion of deacetylnomilinate to nomilin in Citrus limon. Phytochemistry. 1986;25(11):2523-4.

46.

Cárdenas PD, Sonawane PD, Pollier J, Vanden Bossche R, Dewangan V, Weithorn E, Tal L, Meir S, Rogachev I, Malitsky S, Giri AP, Goossens A, Burdman S, Aharoni A. GAME9 regulates the biosynthesis of steroidal alkaloids and upstream isoprenoids in the plant mevalonate pathway. Nat Commun. 2016; 7(1).

47.

Yin J, Li X, Zhan Y, Li Y, Qu Z, Sun L, Wang S, Yang J, Xiao J. Cloning and expression of BpMYC4 and BpbHLH9 genes and the role of BpbHLH9 in triterpenoid synthesis in birch. Bmc Plant Biol. 2017; 17(1).

48.

Ma D, Reichelt M, Yoshida K, Gershenzon J, Constabel CP. Two. R2R3-MYB proteins are broad repressors of flavonoid and phenylpropanoid metabolism in poplar. Plant J. 2018;96(5):949-65.

49.

Jia N, Liu J, Sun Y, Tan P, Cao H, Xie Y, Wen B, Gu T, Liu J, Li M, Huang Y, Lu J, Jin N, Sun L, Xin F, Fan B. Citrus sinensis MYB transcription factors CsMYB330 and CsMYB308 regulate fruit juice sac lignification through finetuning expression of the Cs4CL1 gene. Plant Sci. 2018;277:334-43.

50 .

Livak KJ, Schmittgen TD. Analysis of relative gene expression data using real-time quantitative PCR and the 2 $\Delta \Delta$ CT Method. Methods. 2001;25(4):402-8.

51.

Peng A, Xu L, He Y, Lei T, Yao L, Chen S, Zou X. Efficient production of marker-free transgenic 'Tarocco' blood orange (Citrus sinensis Osbeck) with enhanced resistance to citrus canker using a Cre/loxP site-recombination system. Plant Cell Tissue Organ Cult. 2015;123(1):1-13.

\section{Figures}


a

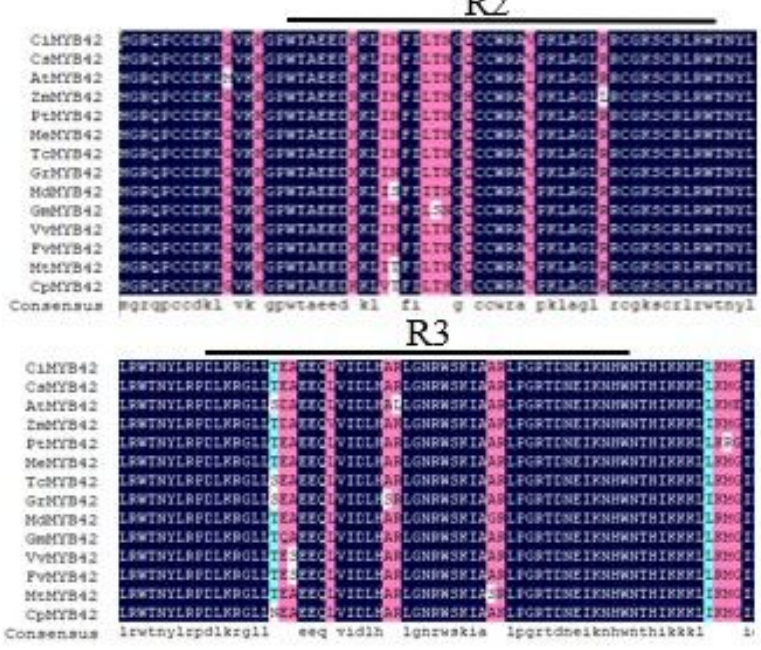

b

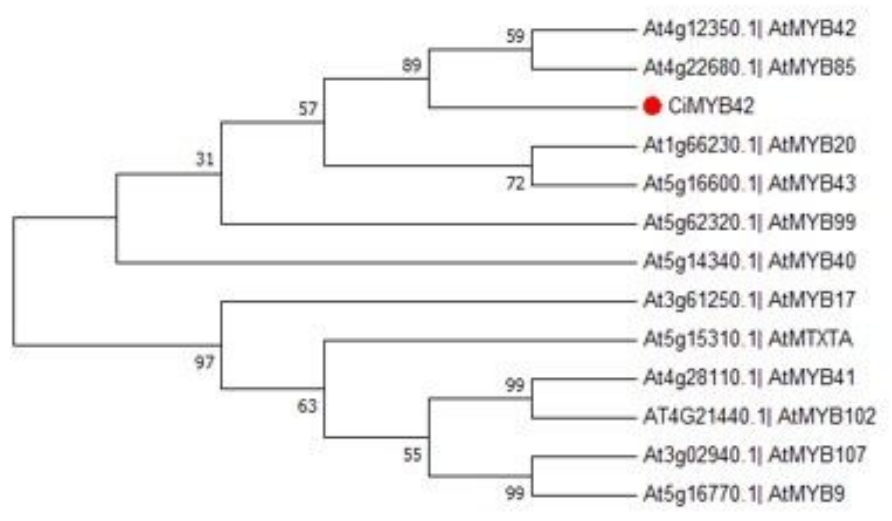

\section{Figure 1}

Amino acid sequence alignment and phylogenetic analysis. (a) Alignment of CiMYB42 and its homologous sequences in other species. The amino acid sequences shown are $\mathbb{C}$. clementina (ciclev10021695m); C. sinensis (Cs5g09410); Arabidopsis thaliana (AT4G12350.1); Zea mays (GRMZM2G104551_T01); Populus trichocarpa (Potri.003G114100.1) $₫$ Manihot esculenta (Manes.02G034300.1); Theobroma cacao (Thecc1EG016054t1); Gossypium raimondii (Gorai.003G168100.1); Malus domestica (MDP0000682032); Glycine max (Glyma.01G211500.1); Vitis vinifera (GSVIVT01004317001); Fragaria vesca subsp. Vesca (mrna32268.1-v1.0hybrid) $₫$ Medicago truncatula (Medtr4g102380.1); Carica papaya (evm.model.supercontig_3.300). (b) Phylogenetic tree of CiMYB42 and R2R3MYB transcription factors from Arabidopsis thaliana. The phylogenetic tree was constructed using MEGA 7.

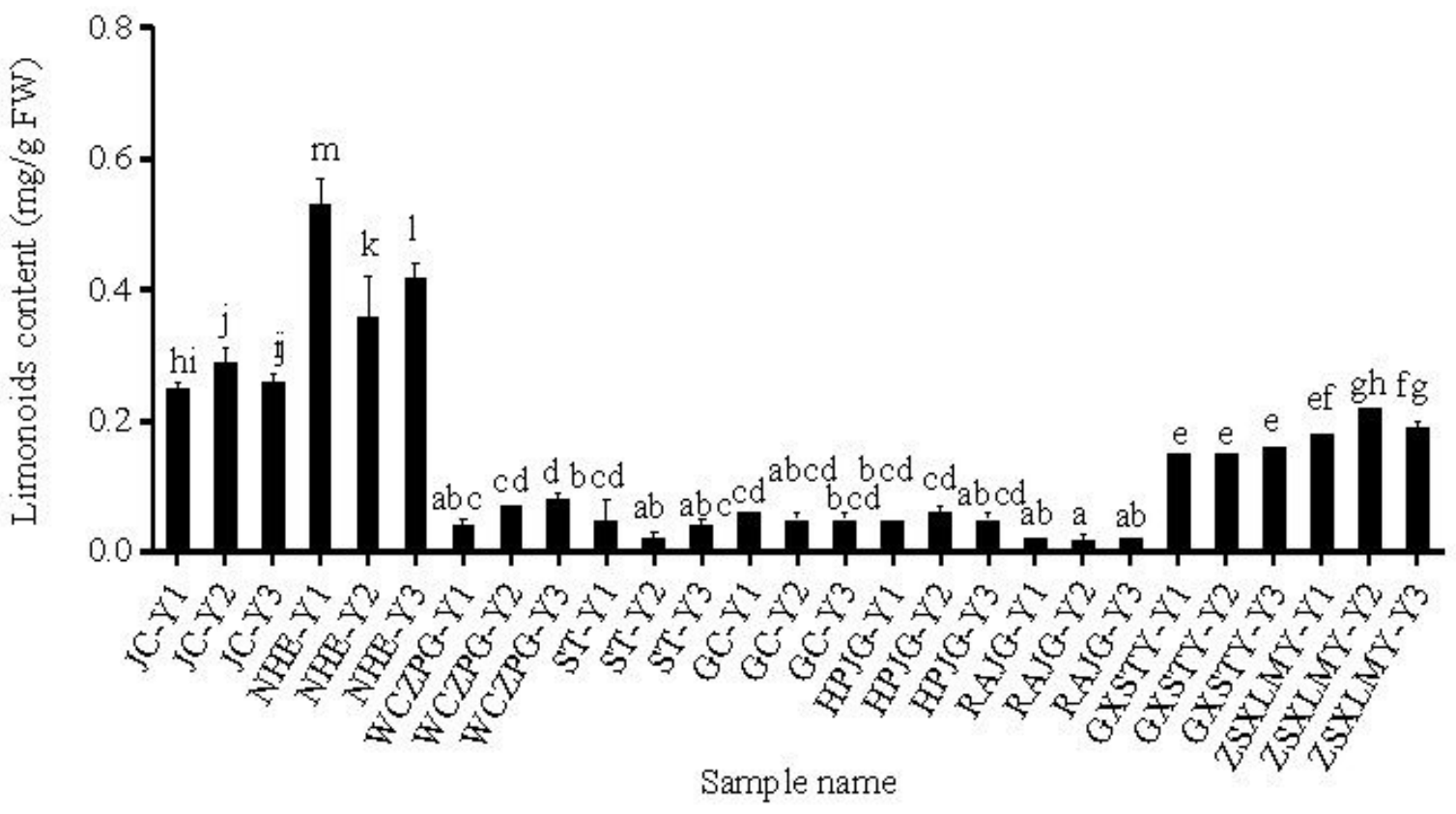

Figure 2 
Limonoids contents in different citrus varieties during leaf development. The abbreviation of samples are as follows: Beibei 447 Jingcheng (JC), Newhall navel orange (NHE), Guangxi Shatian pummelo (GXSTY), Early Siam pummelo (ZSXLMY), Shengtian Satsuma Mandarin (ST), Gongchuan Satsuma Mandarin (GC), Wangcang Zoupi Gan (WCZPG), Huapi kumquat (HPJG), and Rongan kumquat (RAJG), respectively. Y1, Y2 and Y3 are three leaf development stages, respectively.

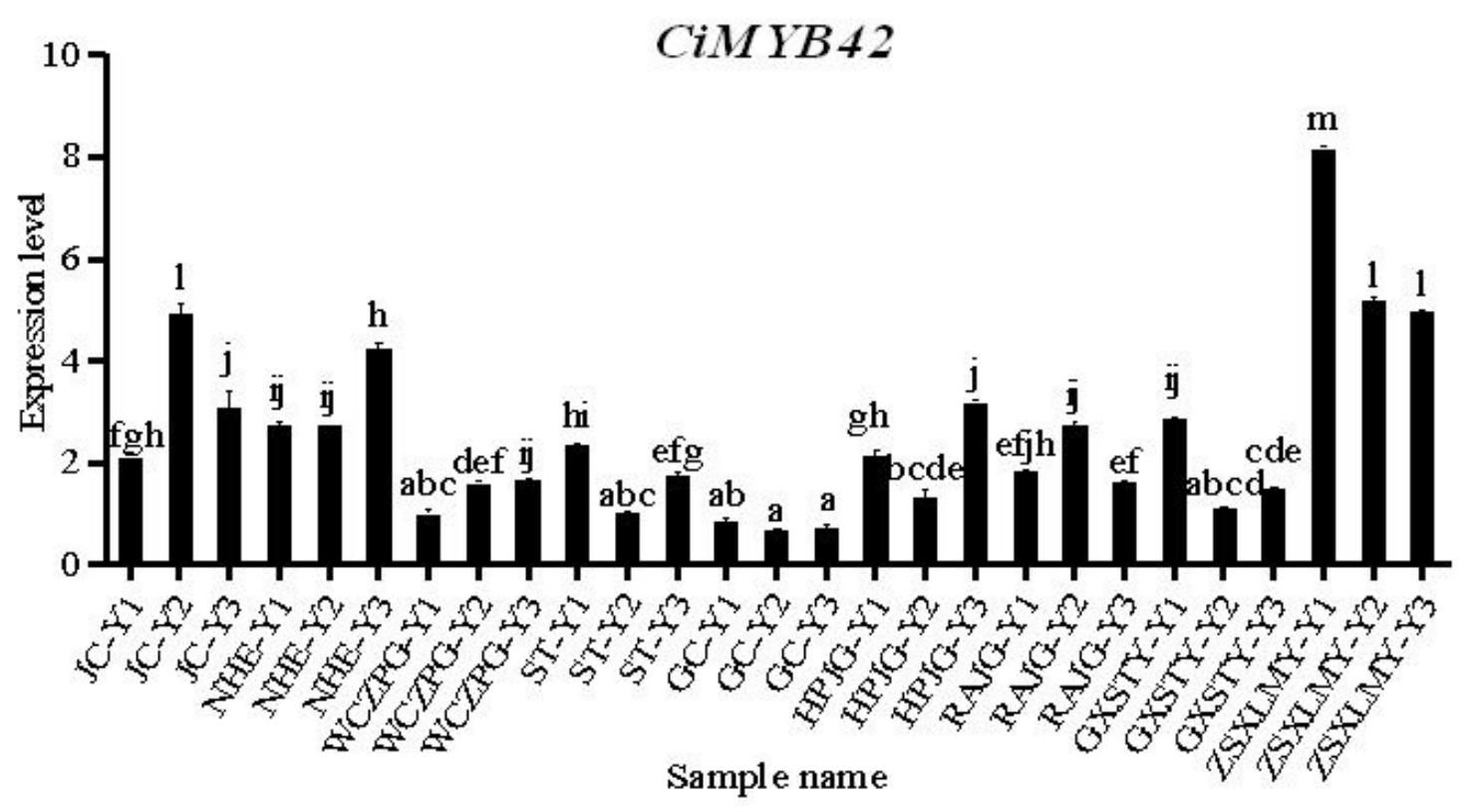

\section{Figure 3}

CiMYB42 expression level in different citrus varieties during leaf development. The sample abbreviation are as follows: Beibei 447 Jingcheng (JC), Newhall navel orange (NHE), Guangxi Shatian pummelo (GXSTY), Early Siam pummelo (ZSXLMY), Shengtian Satsuma Mandarin (ST), Gongchuan Satsuma Mandarin (GC), Wangcang Zoupi Gan (WCZPG), Huapi kumquat (HPJG), and Rongan kumquat (RAJG), respectively. Y1, Y2 and Y3 are three leaf development stages, respectively. 


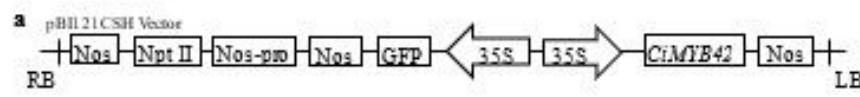

b

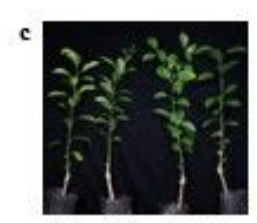

$n x+$ or -2 -...

f
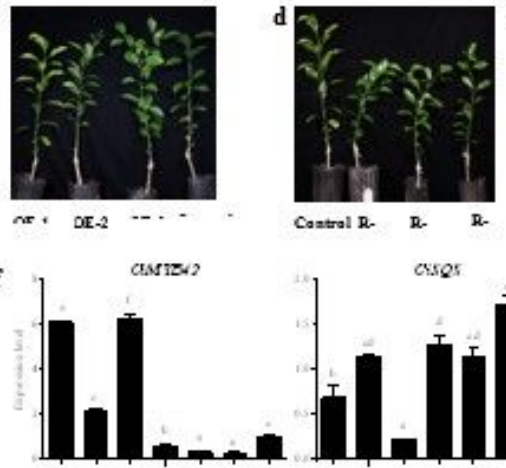

asses

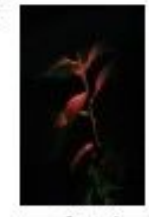

Castral

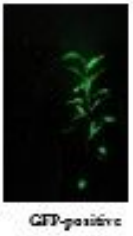

$\operatorname{cose}$

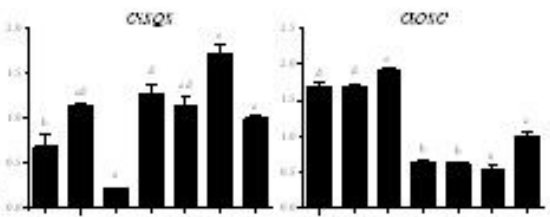

g

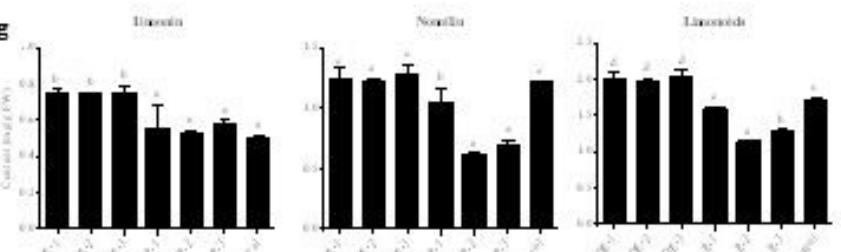

\section{Figure 4}

Analysis of CiMYB42 overexpression lines and RNAi lines. (a) A schematic diagram representing recombinant vector of overexpression; (b) A schematic diagram representing recombinant vector of RNAi; (c) Images of control and CiMYB42-overexpressing transgenic plants; (d) Images of control and CiMYB42-RNAi transgenic plants; (e) Images of control and transgenic positive plants with GFP protein; (f) RT-qPCR analysis of CiMYB42, CiSQS, CiOSC expressions in overexpression and RNAi transgenic lines; (g) Limonin, nomilin and limonoids content in leaves of transgenic plants.

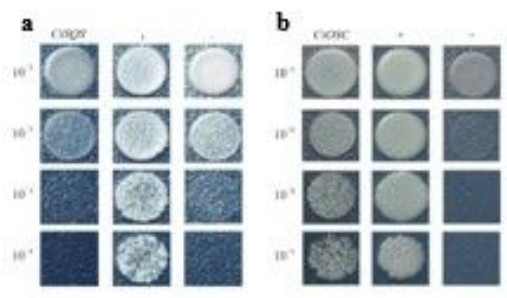

\section{Figure 5}

Identification of interaction between CiMYB42 and promoters of CiSQS and CiOSC with yeast one-hybrid assays. (a) The interaction between CiMYB42 and CiSQS under the condition of 200ng/ml AbA; (b) The interaction between CiMYB42 and CiOSC under the condition of 500ng/ml AbA; (+) Positive control: pGADT7-p53+Y1H [pAbAi-p53]; (-) 
Negative control: pGADT7+Y1H [pAbAi-p53]; the recombinant $\mathrm{Y} 1 \mathrm{H}$ Gold yeast strain were selected by SD/ -leu medium containing optimal AbA concentrations; 10-1, 10-2, 10-3 and10-4 are different dilution ratio.

\section{Supplementary Files}

This is a list of supplementary files associated with this preprint. Click to download.

- Additionalfile1.docx

- Additionalfile2.docx 